\title{
Deep inelastic scattering in the dipole picture at next-to-leading order
}

\section{Ducloue, B.}

2017-11-20

Ducloue , B , Hänninen , H , Lappi , T \& Zhu , Y 2017 , ' Deep inelastic scattering in the dipole picture at next-to-leading order ' , Physical Review D , vol. 96 , no. 9 , 094017 . https://doi.org/10.1103/PhysR

http://hdl.handle.net/10138/233543

https://doi.org/10.1103/PhysRevD.96.094017

other

publishedVersion

Downloaded from Helda, University of Helsinki institutional repository.

This is an electronic reprint of the original article.

This reprint may differ from the original in pagination and typographic detail.

Please cite the original version. 


\title{
Deep inelastic scattering in the dipole picture at next-to-leading order
}

\author{
B. Ducloué, ${ }^{1,2}$ H. Hänninen, ${ }^{1}$ T. Lappi, ${ }^{1,2}$ and Y. Zhu ${ }^{1,2}$ \\ ${ }^{1}$ Department of Physics, P.O. Box 35, 40014 University of Jyväskylä, Finland \\ ${ }^{2}$ Helsinki Institute of Physics, P.O. Box 64, 00014 University of Helsinki, Finland
}

(Received 7 September 2017; published 20 November 2017)

\begin{abstract}
We study quantitatively the importance of the recently derived next-to-leading-order corrections to the deep inelastic scattering structure functions at small $x$ in the dipole formalism. We show that these corrections can be significant and depend on the factorization scheme used to resum large logarithms of energy into renormalization group evolution with the Balitsky-Kovchegov equation. This feature is similar to what has recently been observed for single inclusive forward hadron production. Using a factorization scheme consistent with the one recently proposed for the single inclusive cross section, we show that it is possible to obtain meaningful results for the deep inelastic scattering cross sections.
\end{abstract}

DOI: 10.1103/PhysRevD.96.094017

\section{INTRODUCTION}

At high energy (or equivalently small values of the longitudinal momentum fraction $x$ ), the gluon density in hadrons can become nonperturbatively large; this is the regime of gluon saturation. However, the evolution of this gluon density as a function of the momentum fraction $x$ can still be computed using weak coupling techniques, leading to the Balitsky-Kovchegov (BK) evolution equation $[1,2]$. Knowing the initial gluon density at a given $x=x_{0}$, one can thus evolve it perturbatively to any $x<x_{0}$. This initial condition involves nonperturbative dynamics and needs to be extracted from data, but the evolution equation then gives a first principles prediction for smaller $x$.

The cleanest process to study the partonic structure of hadrons is provided by deep inelastic scattering (DIS). At small $x$, this process is most conveniently understood in the dipole picture, where the scattering is factorized into a QED splitting of the virtual photon into a quark-antiquark dipole and the subsequent QCD interaction of this dipole with the target. Here, the BK equation describes the dependence of the dipole-target scattering amplitude on the collision energy. Several groups have been able to obtain satisfactory fits to HERA DIS data in the leading-order dipole picture, using the BK equation with running coupling corrections (see for example Refs. [3,4]). To advance the saturation formalism to next-to-leading order (NLO), two key ingredients are needed: the NLO BK equation and the processdependent NLO impact factors. In addition to many recent methodological developments for these higher-order calculations (see e.g. Refs. [5,6]), progress has been made in both of these directions. The NLO corrections to the BK equation have been computed in Ref. [7] and evaluated numerically in Ref. [8], where it was shown that they can lead to unphysical results. This problem has been subsequently solved by resumming classes of large logarithms [9-11], indeed leading to reasonable results [12].

Concerning impact factors, most of the recent work has concentrated on the NLO corrections to single inclusive forward hadron production. The impact factor for this process has been known for some time [13,14], but the first numerical implementation of these expressions showed that they can make the cross section negative when the transverse momentum of the produced hadron is of the order of a few $\mathrm{GeV}$ [15]. Several works have been devoted to solving this issue [16-20], and recently a new proposed formulation of the NLO cross section [21] was shown to lead to physical results [22], albeit with a remaining issue concerning the best way to implement a running QCD coupling constant.

Also, the impact factor for DIS in the dipole picture has been studied in several papers [23-26]. However, the full expressions in the mixed space representation (longitudinal momentum, but transverse coordinate) that are most naturally combined with BK evolution have only become available more recently $[27,28]$. For a practical implementation of these results, it is essential to match the impact factor calculation with the evolution equation in the correct way, i.e. to factorize the leading high energy logarithms into the high energy evolution. As we shall discuss below, the situation here is very analogous to that of single inclusive particle production.

The main purpose of this paper is twofold. We first want to study the importance of the NLO corrections to have a first estimate of the stability of the perturbative expansion for this quantity. Second, we want to develop a good factorization procedure for matching the renormalization group evolution with the previous calculation of the impact factor. Both of these are prerequisites for a description of experimental data, which will be pursued in a continuation of this work. Our focus in this paper is to demonstrate the feasibility of the factorization scheme and study the general characteristics of the NLO corrections to the cross sections. A full NLO calculation will additionally require including an NLO evolution equation. In this paper, we shall first, in Sec. II, briefly present the NLO impact factor as calculated in Refs. [27,28]. We shall then, in Sec. III, quantify the 
effects of the NLO corrections for the $Q^{2}$ - and $x_{B j^{-}}$ dependence of the transverse and longitudinal DIS cross sections.

\section{IMPACT FACTOR}

In the dipole framework, the interaction of a virtual photon with the proton in DIS is factorized as the scattering of a quark-antiquark dipole with the proton. At leading order, the expressions for the cross sections of transversally or longitudinally polarized virtual photons $\sigma_{L, T}$ read

$$
\begin{aligned}
\sigma_{L, T}^{\mathrm{LO}}\left(x_{B j}, Q^{2}\right)= & 4 N_{\mathrm{c}} \alpha_{e m} \sum_{f} e_{f}^{2} \int_{0}^{1} \mathrm{~d} z_{1} \\
& \times \int_{\mathbf{x}_{0}, \mathbf{x}_{1}} \mathcal{K}_{L, T}^{\mathrm{LO}}\left(z_{1}, \mathbf{x}_{0}, \mathbf{x}_{1}, x_{B j}\right),
\end{aligned}
$$

with the shorthand $\int_{\mathbf{x}_{0}}=\int \frac{\mathrm{d}^{2} \mathbf{x}_{0}}{2 \pi}$. The integrands are given by the squares of the light cone wave functions for the $\gamma^{*} \rightarrow q \bar{q}$ splitting and the scattering amplitudes for the $q \bar{q}$ dipole to scatter off the target

$$
\begin{aligned}
\mathcal{K}_{L}^{\mathrm{LO}}\left(z_{1}, \mathbf{x}_{0}, \mathbf{x}_{1}, X\right)= & 4 Q^{2} z_{1}^{2}\left(1-z_{1}\right)^{2} \\
& \times K_{0}^{2}\left(Q X_{2}\right)\left(1-S_{01}(X)\right), \\
\mathcal{K}_{T}^{\mathrm{LO}}\left(z_{1}, \mathbf{x}_{0}, \mathbf{x}_{1}, X\right)= & Q^{2} z_{1}\left(1-z_{1}\right)\left(z_{1}^{2}+\left(1-z_{1}\right)^{2}\right) \\
& \times K_{1}^{2}\left(Q X_{2}\right)\left(1-S_{01}(X)\right),
\end{aligned}
$$

for the longitudinal $(L)$ and transverse $(T)$ polarized virtual photons respectively. Here, the argument of the Bessel functions, related to the lifetime of the $q \bar{q}$-fluctuation, is $X_{2}^{2}=z_{1}\left(1-z_{1}\right) \mathbf{x}_{01}^{2}$. The scattering amplitude of the dipole is given, in the Color Glass Condensate picture, by the two point function of a correlator of Wilson lines, namely

$$
\begin{aligned}
S_{01}(X) & \equiv S\left(\mathbf{x}_{01}=\mathbf{x}_{0}-\mathbf{x}_{1}, X\right) \\
& =\left\langle\frac{1}{N_{\mathrm{c}}} \operatorname{Tr} U\left(\mathbf{x}_{0}\right) U^{\dagger}\left(\mathbf{x}_{1}\right)\right\rangle_{X},
\end{aligned}
$$

where we denote by $X$ the momentum fraction (corresponding to the evolution variable in the $\mathrm{BK}$ equation $y=\ln 1 / X)$ at which the Wilson line correlator is to be evaluated.

The NLO corrections to these expressions have been computed in Refs. [27,28]. They involve two kinds of terms: the one loop corrections to the $q \bar{q}$-state and a new $q \bar{q} g$-component in the $\gamma^{*}$ Fock state. Following the general idea exposed in Ref. [21] for single inclusive hadron production, we write the (unsubtracted) NLO cross sections as

$$
\sigma_{L, T}^{\mathrm{NLO}}=\sigma_{L, T}^{(0)}+\sigma_{L, T}^{q g}+\sigma_{L, T}^{\mathrm{dip}}
$$

In this expression, the first term corresponds to the lowestorder contribution with an unevolved target (i.e. evaluated at the rapidity $X=x_{0}$ ). The terms proportional to $\alpha_{\mathrm{s}}$ have been organized into two parts. First, the gluon contribution $\sigma_{L, T}^{q g}$ includes all the real contributions (with a gluon emitted into the final state) and a subset of the virtual corrections that need to be combined with the real corrections to cancel any ultraviolet or collinear divergences. The dipole contribution $\sigma_{L, T}^{\mathrm{dip}}$ contains the rest of the virtual corrections. The separation between these two terms is not unique, but the sum of the two is fully determined by the NLO calculation. The expressions for these terms can be written as

$$
\begin{aligned}
\sigma_{L, T}^{q g}= & 8 N_{\mathrm{c}} \alpha_{e m} \frac{\alpha_{s} C_{\mathrm{F}}}{\pi} \sum_{f} e_{f}^{2} \int_{0}^{1} \mathrm{~d} z_{1} \int^{1-z_{1}} \frac{\mathrm{d} z_{2}}{z_{2}} \\
& \times \int_{\mathbf{x}_{0}, \mathbf{x}_{1}, \mathbf{x}_{2}} \mathcal{K}_{L, T}^{\mathrm{NLO}}\left(z_{1}, z_{2}, \mathbf{x}_{0}, \mathbf{x}_{1}, \mathbf{x}_{2}, X\left(z_{2}\right)\right), \\
\sigma_{L, T}^{\mathrm{dip}}= & 4 N_{\mathrm{c}} \alpha_{e m} \frac{\alpha_{s} C_{\mathrm{F}}}{\pi} \sum_{f} e_{f}^{2} \int_{0}^{1} \mathrm{~d} z_{1} \\
& \times \int_{\mathbf{x}_{0}, \mathbf{x}_{1}} \mathcal{K}_{L, T}^{\mathrm{LO}}\left(z_{1}, \mathbf{x}_{0}, \mathbf{x}_{1}, X^{\mathrm{dip}}\right) \\
& \times\left[\frac{1}{2} \ln ^{2}\left(\frac{z_{1}}{1-z_{1}}\right)-\frac{\pi^{2}}{6}+\frac{5}{2}\right],
\end{aligned}
$$

with

$$
\begin{aligned}
\mathcal{K}_{L}^{\mathrm{NLO}}\left(z_{1}, z_{2}, \mathbf{x}_{0}, \mathbf{x}_{1}, \mathbf{x}_{2}, X\right)=4 Q^{2} z_{1}^{2}\left(1-z_{1}\right)^{2} \\
\quad \times\left\{P\left(\frac{z_{2}}{1-z_{1}}\right) \frac{\mathbf{x}_{20}}{\mathbf{x}_{20}^{2}} \cdot\left(\frac{\mathbf{x}_{20}}{\mathbf{x}_{20}^{2}}-\frac{\mathbf{x}_{21}}{\mathbf{x}_{21}^{2}}\right)\right. \\
\quad \times\left[K_{0}^{2}\left(Q X_{3}\right)\left(1-S_{012}(X)\right)-\left(\mathbf{x}_{2} \rightarrow \mathbf{x}_{0}\right)\right] \\
\left.\quad+\left(\frac{z_{2}}{1-z_{1}}\right)^{2} \frac{\mathbf{x}_{20} \cdot \mathbf{x}_{21}}{\mathbf{x}_{20}^{2} \mathbf{x}_{21}^{2}} K_{0}^{2}\left(Q X_{3}\right)\left(1-S_{012}(X)\right)\right\}, \quad(8) \\
\mathcal{K}_{T}^{\mathrm{NLO}}\left(z_{1}, z_{2}, \mathbf{x}_{0}, \mathbf{x}_{1}, \mathbf{x}_{2}, X\right)=Q^{2} z_{1}\left(1-z_{1}\right) \\
\quad \times\left\{P\left(\frac{z_{2}}{1-z_{1}}\right)\left(z_{1}^{2}+\left(1-z_{1}\right)^{2}\right) \frac{\mathbf{x}_{20}}{\mathbf{x}_{20}^{2}}\right. \\
\quad \cdot\left(\frac{\mathbf{x}_{20}}{\mathbf{x}_{20}^{2}}-\frac{\mathbf{x}_{21}}{\mathbf{x}_{21}^{2}}\right)\left[K_{1}^{2}\left(Q X_{3}\right)\left(1-S_{012}(X)\right)-\left(\mathbf{x}_{2} \rightarrow \mathbf{x}_{0}\right)\right] \\
+\left(\frac{z_{2}}{1-z_{1}}\right)^{2}\left[\left(z_{1}^{2}+\left(1-z_{1}\right)^{2}\right) \frac{\mathbf{x}_{20} \cdot \mathbf{x}_{21}}{\mathbf{x}_{20}^{2} \mathbf{x}_{21}^{2}}\right. \\
\left.\left.+2 z_{0} z_{1} \frac{\mathbf{x}_{20} \cdot \mathbf{x}_{21}}{\mathbf{x}_{20}^{2} X_{3}^{2}}-\frac{z_{0}\left(z_{1}+z_{2}\right)}{X_{3}^{2}}\right] K_{1}^{2}\left(Q X_{3}\right)\left(1-S_{012}(X)\right)\right\} .
\end{aligned}
$$

Here, the longitudinal momentum fractions of the quark, antiquark, and gluon are denoted as $z_{0}, z_{1}, z_{2}$ with 
$z_{0}+z_{1}+z_{2}=1$. The argument of the Bessel functions, related to the lifetime of the $q \bar{q} g$-fluctuation, is $X_{3}^{2}=$ $z_{0} z_{1} \mathbf{x}_{01}^{2}+z_{0} z_{2} \mathbf{x}_{20}^{2}+z_{2} z_{1} \mathbf{x}_{21}^{2}, \quad P(z)=1+(1-z)^{2}, \quad$ and the Wilson line operator corresponding to the scattering of the $q \bar{q} g$-state is

$$
S_{012}(X)=\frac{N_{\mathrm{c}}}{2 C_{\mathrm{F}}}\left(S_{02}(X) S_{21}(X)-\frac{1}{N_{\mathrm{c}}^{2}} S_{01}(X)\right) .
$$

It is important to note that, because the functions $\mathcal{K}_{L, T}^{\mathrm{NLO}}\left(z_{1}, z_{2}, \mathbf{x}_{0}, \mathbf{x}_{1}, \mathbf{x}_{2}, X\right)$ approach a nonzero value when $z_{2} \rightarrow 0$ at fixed $X$, the integral over $z_{2}$ in $\sigma_{L, T}^{q g}$ produces a large logarithm which should be resummed in the BK evolution of the target. We will do this using the same procedure introduced in Refs. [9,21] and demonstrated in Ref. [22] for the case of single inclusive particle production in forward proton-nucleus collisions. Note that, similarly to the " $C_{\mathrm{F}}$-term" in the case of the single inclusive cross section, the "dipole" term does not generate such a large logarithmic contribution and therefore does not contribute to the BK evolution.

The starting point of the BK-factorization procedure is to identify the first term in Eq. (5) as the initial condition for the BK evolution with the longitudinal momentum fraction $x_{0} \sim 0.01$, i.e.

$\sigma_{L, T}^{\mathrm{IC}}=4 N_{\mathrm{c}} \alpha_{e m} \sum_{f} e_{f}^{2} \int_{0}^{1} \mathrm{~d} z_{1} \int_{\mathbf{x}_{0}, \mathbf{x}_{1}} \mathcal{K}_{L, T}^{\mathrm{LO}}\left(z_{1}, \mathbf{x}_{0}, \mathbf{x}_{1}, x_{0}\right)$.

As discussed in great detail in Refs. [9,21], the essential feature required for a stable perturbative expansion is that the dipole correlators in $\sigma_{L, T}^{q g}$ must be evaluated at a rapidity scale that depends on the longitudinal momentum of the emitted gluon, i.e. $z_{2}$. Here, there are several different possibilities, which are all equivalent at the leading logarithmic level. At NLO accuracy, the different schemes lead to different expressions which are in principle equivalent, but more naturally lend themselves to different approximations.

The choice advocated in Ref. [9] is to consistently use the probe longitudinal momentum $k^{+}$as the evolution variable, sometimes referred to as "probe evolution." In this case, the evolution rapidity is by definition $y=\ln 1 / z_{2}+y_{0}$ with some constant $y_{0}$ used to make $y=0$ correspond to the initial condition for the evolution. To determine the lower integration limit for $z_{2}$ in this scheme, we have to compare the longitudinal momentum of the emitted soft gluon $z_{2} q^{+}$to momentum scales in the target. The typical target hadronic momentum scale is given by $P^{+}=Q_{0}^{2} /\left(2 P^{-}\right)$, where $Q_{0}$ is some hadronic low transverse momentum scale and the total target light cone energy $P^{-}$is obtained from the total centerof-mass energy of the $\gamma^{*}$-target system by $W^{2}=2 q^{+} P^{-}$. For the eikonal approximation to be valid, we require that the probe gluon momentum is larger than the target momentum scale by a large factor $1 / x_{0}$, i.e. $z_{2} q^{+}>\left(1 / x_{0}\right) P^{+}$. This translates, using $x_{B j} \approx Q^{2} / W^{2}$, into an integration limit, $z_{2}>\left(x_{B j} / x_{0}\right)\left(Q_{0}^{2} / Q^{2}\right)$. If now the soft gluon has a transverse momentum $k_{\perp}$, the light cone energy required from the target to put the $q \bar{q} g$-state on shell is $\Delta k^{-} \gtrsim k_{\perp}^{2} /\left(2 z_{2} q^{+}\right)$. The limit on $z_{2}$ means that we allow the $\gamma^{*}$ system to take a fraction $\Delta k^{-} / P^{-} \lesssim x_{0}\left(k_{\perp}^{2} / Q_{0}^{2}\right)$ of the target light cone energy. If the typical gluon $k_{\perp}$ is at the hadronic scale $Q_{0}$, this is indeed the limit $\Delta k^{-} / P^{-}<x_{0}$ that we would want for the fraction of the target light cone energy. However, the contribution from $k_{\perp}^{2} \sim Q^{2} \gg Q_{0}^{2}$ goes to larger values of the target momentum fraction $\Delta k^{-} / P^{-}$than we would want. This can generally be expected to be a problem that must be corrected by imposing an additional "kinematical constraint" on the evolution equation [9,29] and on the impact factor $[17,19,20]$.

The other option to probe evolution is to take the view that the evolution variable should always be the target momentum fraction, i.e. the fraction of the target light cone energy $X=\Delta k^{-} / P^{-}$. Keeping this momentum fraction small, $X<x_{0}$, removes the need for an additional kinematical constraint, significantly simplifying the evolution equation. On the other hand, using $\Delta k^{-} / P^{-}$as the evolution variable adds the significant complication that this momentum fraction depends on the transverse momentum of the gluon, $X\left(z_{2}\right) \approx k_{\perp}^{2} /\left(z_{2} W^{2}\right)$, and when $z_{2}$ is not very small also on the momenta of the quark and antiquark. This makes it difficult to implement a light cone energy factorization scale or evolution variable exactly. Parametrically, the transverse momentum $k_{\perp}$ can range from a hadronic scale $Q_{0}$ to the hard scale $Q$. If one estimates the typical target momentum fraction $\Delta k^{-}$ assuming that the typical gluon transverse momentum is at the hadronic scale $k_{\perp}^{2} \sim Q_{0}^{2}$, one recovers the same limit $z_{2}>\left(x_{B j} / x_{0}\right)\left(Q_{0}^{2} / Q^{2}\right)$ as argued from using $k^{+}$as the factorization variable. In contrast, the argument used in the recent work on single inclusive particle production in proton-nucleus collisions $[21,22]$ was that, at least in that case, the typical transverse momentum of the gluon in the impact factor is in fact the hard scale of the process $k_{\perp} \sim Q$. Assuming that this is the case also for DIS means that one should restrict the integrals to a smaller phase space $z_{2}>\left(x_{B j} / x_{0}\right)$. The latter is the limit that we will use in this work. In terms of the $k^{+}$-momentum, this limit corresponds to the emitted gluon having longitudinal momentum $z_{2} q^{+} \gtrsim\left(Q^{2} / Q_{0}^{2}\right)\left(1 / x_{0}\right) P^{+}$instead of the $z_{2} q^{+}>\left(1 / x_{0}\right) P^{+}$that one would use in the factorization scheme with $k^{+}$. This approximation leads to a rather simple formulation for the cross section. Improving the accuracy would require including the additional phase space $\left(x_{B j} / x_{0}\right)\left(Q_{0}^{2} / Q^{2}\right)<z_{2}<\left(x_{B j} / x_{0}\right)$ in the cross section on one hand but cutting out the large logarithmic increase from this region by using a kinematical constraint 
in the evolution equation, as advocated e.g. in Refs. $[9,27,28]$. Due to the considerably increased complication of this formulation, we will defer studying this alternative to future work.

To summarize, in this paper, we will follow the choice made for single inclusive particle production in protonnucleus collisions in Refs. [21,22] and choose the target momentum fraction as the evolution variable, supplemented with the assumption that all transverse momenta are of the order $Q$. Thus, we take $X\left(z_{2}\right)=x_{B j} / z_{2}$ and set the kinematical limit by requiring $X\left(z_{2}\right)<x_{0}$, i.e. $z_{2}>x_{B j} / x_{0}$. Implementing this limit, we can now complete the "unsubtracted" form of the cross section (5) with the lower integration limit in $z_{2}$ as

$$
\sigma_{L, T}^{\mathrm{NLO}}=\sigma_{L, T}^{\mathrm{IC}}+\sigma_{L, T}^{q g, \text { unsub }}+\sigma_{L, T}^{\operatorname{dip}},
$$

with

$$
\begin{aligned}
\sigma_{L, T}^{q g, \text { unsub }}= & 8 N_{\mathrm{c}} \alpha_{e m} \frac{\alpha_{s} C_{\mathrm{F}}}{\pi} \sum_{f} e_{f}^{2} \int_{0}^{1} \mathrm{~d} z_{1} \int_{x_{B j} / x_{0}}^{1-z_{1}} \frac{\mathrm{d} z_{2}}{z_{2}} \\
& \times \int_{\mathbf{x}_{0}, \mathbf{x}_{1}, \mathbf{x}_{2}} \mathcal{K}_{L, T}^{\mathrm{NLO}}\left(z_{1}, z_{2}, \mathbf{x}_{0}, \mathbf{x}_{1}, \mathbf{x}_{2}, X\left(z_{2}\right)\right) .
\end{aligned}
$$

We then note that taking $z_{2}=0$ as the explicit $z_{2}$-argument in $\mathcal{K}_{L, T}^{\mathrm{NLO}}$ [but not in the implicit dependence through $X\left(z_{2}\right)$ ] leads to an integral version of the BK equation. Using this, we can also rewrite Eq. (12) in a form that involves the leading-order cross sections with BK-evolved dipole operators evaluated at the scale $x_{B j}$ instead of $x_{0}$. The result is a strictly equivalent "subtracted" form of the cross section

$$
\sigma_{L, T}^{\mathrm{NLO}}=\sigma_{L, T}^{\mathrm{LO}}+\sigma_{L, T}^{q g, \mathrm{sub}}+\sigma_{L, T}^{\mathrm{dip}},
$$

where $\sigma_{L, T}^{\mathrm{LO}}$ is the well-known leading-order expression (1) and

$$
\begin{aligned}
\sigma_{L, T}^{q g, \mathrm{sub}}= & 8 N_{\mathrm{c}} \alpha_{e m} \frac{\alpha_{s} C_{\mathrm{F}}}{\pi} \sum_{f} e_{f}^{2} \int_{0}^{1} \mathrm{~d} z_{1} \int_{x_{B j} / x_{0}}^{1} \frac{\mathrm{d} z_{2}}{z_{2}} \\
& \times \int_{\mathbf{x}_{0}, \mathbf{x}_{1}, \mathbf{x}_{2}}\left[\theta\left(1-z_{1}-z_{2}\right)\right. \\
& \times \mathcal{K}_{L, T}^{\mathrm{NLO}}\left(z_{1}, z_{2}, \mathbf{x}_{0}, \mathbf{x}_{1}, \mathbf{x}_{2}, X\left(z_{2}\right)\right) \\
& \left.-\mathcal{K}_{L, T}^{\mathrm{NLO}}\left(z_{1}, 0, \mathbf{x}_{0}, \mathbf{x}_{1}, \mathbf{x}_{2}, X\left(z_{2}\right)\right)\right] .
\end{aligned}
$$

Contrary to $\sigma_{L, T}^{q g}$, the dipole term $\sigma_{L, T}^{\mathrm{dip}}$ is not associated with the rapidity evolution of the target, and thus the rapidity scale of the dipole operators in this term is left unspecified. As presented in Refs. [27,28], this term is already integrated over $z_{2}$. Therefore, it is not possible to evaluate the dipole operators in this term at the same scale $X\left(z_{2}\right)=x_{B j} / z_{2}$ as in $\sigma_{L, T}^{q g}$, which would arguably be the most natural thing to do. Here, we will evaluate this term at $X^{\text {dip }}=x_{B j}$ since the integrand vanishes when $z_{2} \rightarrow 0$, and therefore one can expect the integral to be dominated by the region where $z_{2}$ is close to 1 . Note, however, that the difference between $X=x_{B j} / z_{2}$ and $X=x_{B j}$, while formally subleading for the dipole term, could be numerically important, as is the case for the analogous $C_{\mathrm{F}}$-terms in single inclusive particle production [22].

To obtain the previous expressions, we followed closely the original idea of Ref. [21], which was shown in Ref. [22] to lead to reasonable numerical results for single inclusive particle production at all transverse momenta. Bear in mind that the two expressions in Eqs. (12) and (14) are completely equivalent and are related through the BK evolution equation. In the following, it will also be interesting to compare the results obtained in this formulation with what we denote here as the " $x_{B j}$-subtraction" scheme, which is expressed as

$$
\sigma_{L, T}^{\mathrm{NLO}, x_{B j}-\mathrm{sub}}=\sigma_{L, T}^{\mathrm{LO}}+\sigma_{L, T}^{q g, \mathrm{sub}^{*}}+\sigma_{L, T}^{\mathrm{dip}},
$$

where $\sigma_{L, T}^{q g, \text { sub }^{*}}$ is an approximation of Eq. (15) by using $X\left(z_{2}\right)=x_{B j}$ and taking the limit $x_{B j} / x_{0} \rightarrow 0$ in the lower limit of the integral over $z_{2}$. This is the analog of what was denoted in Refs. [20,22] as the "CXY" subtraction scheme after the authors of $[13,14]$ for the case of single inclusive particle production, which is formally equivalent at this order of perturbation theory, but leads to problematic results for high momentum scales.

\section{NUMERICAL RESULTS}

Since we do not consider a possible impact parameter dependence of the dipole correlators, one of the coordinate integrals in the expressions shown in the previous section is trivial and leads to a factor corresponding to the target transverse area, denoted as $\sigma_{0} / 2$. This quantity is usually determined by a fit to data, such as in Refs. [3,4]. Performing such a fit goes well beyond the scope of the present work; therefore, for simplicity, we leave out this overall normalization factor and present results for $F_{L, T} / \frac{\sigma_{0}}{2}$, where the structure functions $F_{L, T}$ are defined as

$$
F_{L, T}\left(x_{B j}, Q^{2}\right)=\frac{Q^{2}}{4 \pi^{2} \alpha_{\mathrm{em}}} \sigma_{L, T}\left(x_{B j}, Q^{2}\right) .
$$

We first focus on the fixed coupling case, using $\alpha_{\mathrm{s}}=0.2$ both when evaluating the NLO cross section and when solving the leading-order Balitsky-Kovchegov equation. Note that for the factorization scheme to be consistent both the cross section calculation and the BK equation need to have the same coupling constant. For the BK equation, we use a McLerran-Venugopalan initial condition [30]

$$
S\left(\mathbf{r}, x_{0}\right)=\exp \left[-\frac{\mathbf{r}^{2} Q_{\mathrm{s}, 0}^{2}}{4} \ln \left(\frac{1}{|\mathbf{r}| \Lambda_{\mathrm{QCD}}}+e\right)\right],
$$

where we take $Q_{\mathrm{s}, 0}^{2}=0.2 \mathrm{GeV}^{2}$ and $\Lambda_{\mathrm{QCD}}=0.241 \mathrm{GeV}$. 

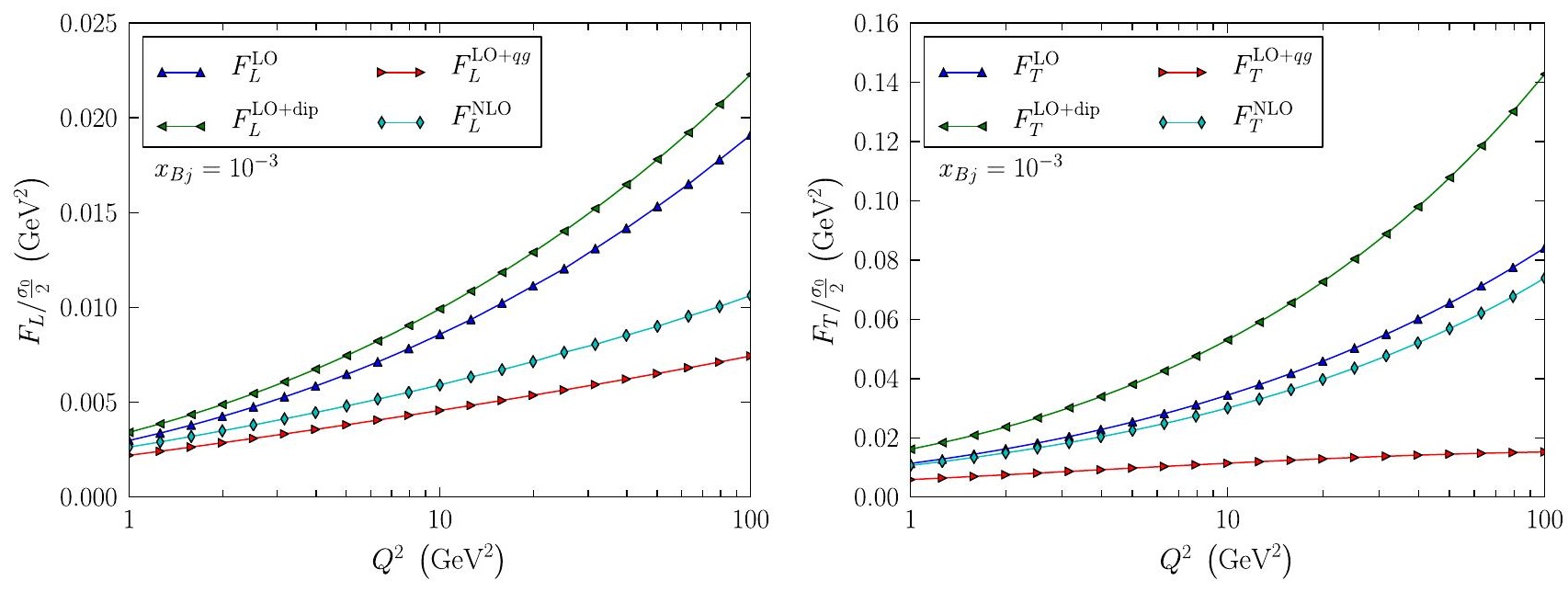

FIG. 1. LO and NLO contributions to $F_{L}$ (left) and $F_{T}$ (right) as a function of $Q^{2}$ at $x_{B j}=10^{-3}$ with $\alpha_{\mathrm{S}}=0.2$.

In Fig. 1, we show the importance of the NLO corrections $\sigma^{\text {dip }}$ and $\sigma^{q g}$ to $F_{L}$ and $F_{T}$ as a function of $Q^{2}$ at $x_{B j}=10^{-3}$. In both the longitudinal and transverse cases, the sign of these corrections is the same: the dipole contribution is positive, which can be understood from Eq. (7), while the $q g$ contribution is negative. Because the second correction is larger in magnitude than the first one, the total NLO cross section is smaller than the leadingorder (LO) one.

In Fig. 2, we show how these results change if we use the approximate $x_{B j}$-subtraction in Eq. (16) for the $q g$-term. This term is still negative and has a larger magnitude, especially at large $Q$, which makes the whole NLO cross section negative for $Q^{2} \gtrsim 10 \mathrm{GeV}^{2}$, both in the longitudinal and transverse cases. Therefore, approximating Eq. (14) by Eq. (16), while in principle justified in a weak coupling sense, has in fact a large effect in this region and can lead to unphysical results. A similar behavior was observed in single inclusive particle production at large transverse momenta [22]. This shows that to get meaningful results one should really use the factorization procedure in Eq. (12) or equivalently Eq. (14), which we will do for the rest of this paper.

We also show in Figs. 3 and 4 the $x_{B j}$-dependence of the different NLO contributions to $F_{L}$ and $F_{T}$ for fixed $Q^{2}=1$ and $50 \mathrm{GeV}^{2}$. These plots show a change of behavior: at small $x_{B j}$, the NLO cross section is smaller than the LO one, while it becomes larger when $x_{B j}$ approaches $x_{0}$. The reason is the following: as explained previously, the dipole NLO correction is always positive. In addition, as can be seen from Eq. (13), the $q g$-part is 0 at $x_{B j}=x_{0}$ since the $z_{2}$-integration range vanishes. Therefore, the NLO cross section is the sum of the leading-order one and a positive correction, i.e. always larger than the leading-order one. This is related to the reason why, as explained in the previous section, we would prefer to use an expression of
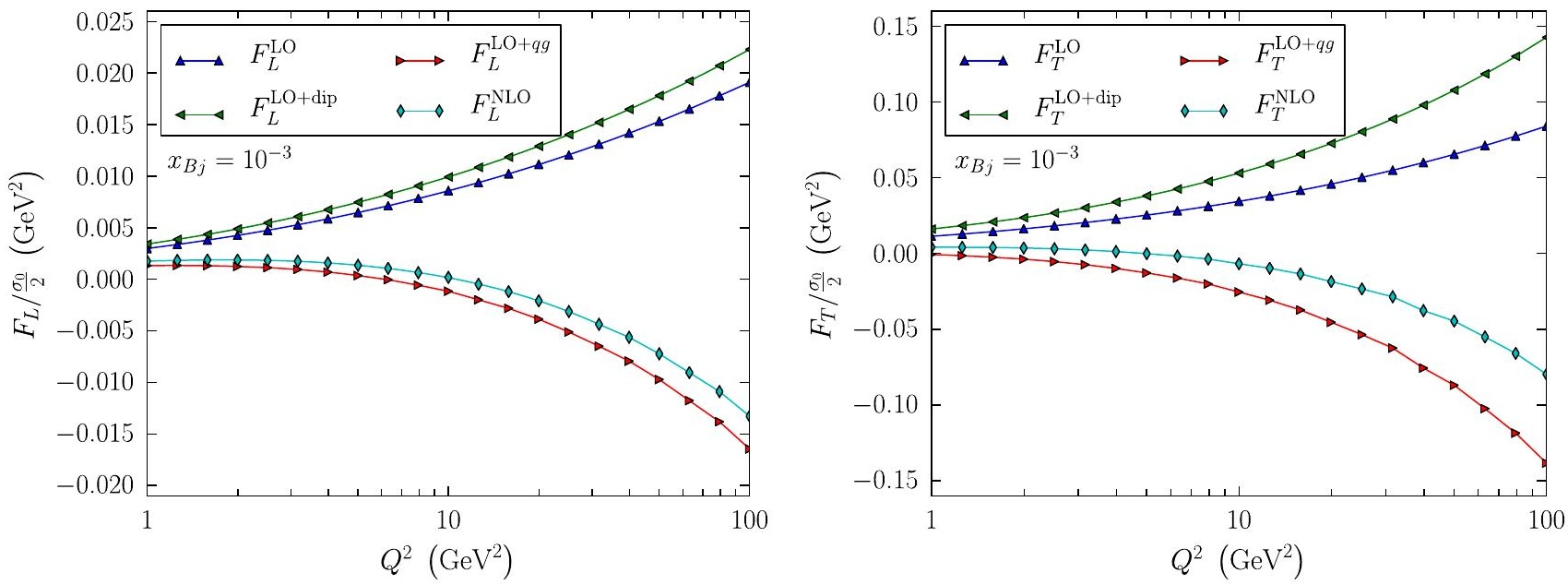

FIG. 2. LO and NLO contributions to $F_{L}$ (left) and $F_{T}$ (right) as a function of $Q^{2}$ at $x_{B j}=10^{-3}$ with $\alpha_{\mathrm{s}}=0.2$ and using the $x_{B j^{-}}$ subtraction procedure. 

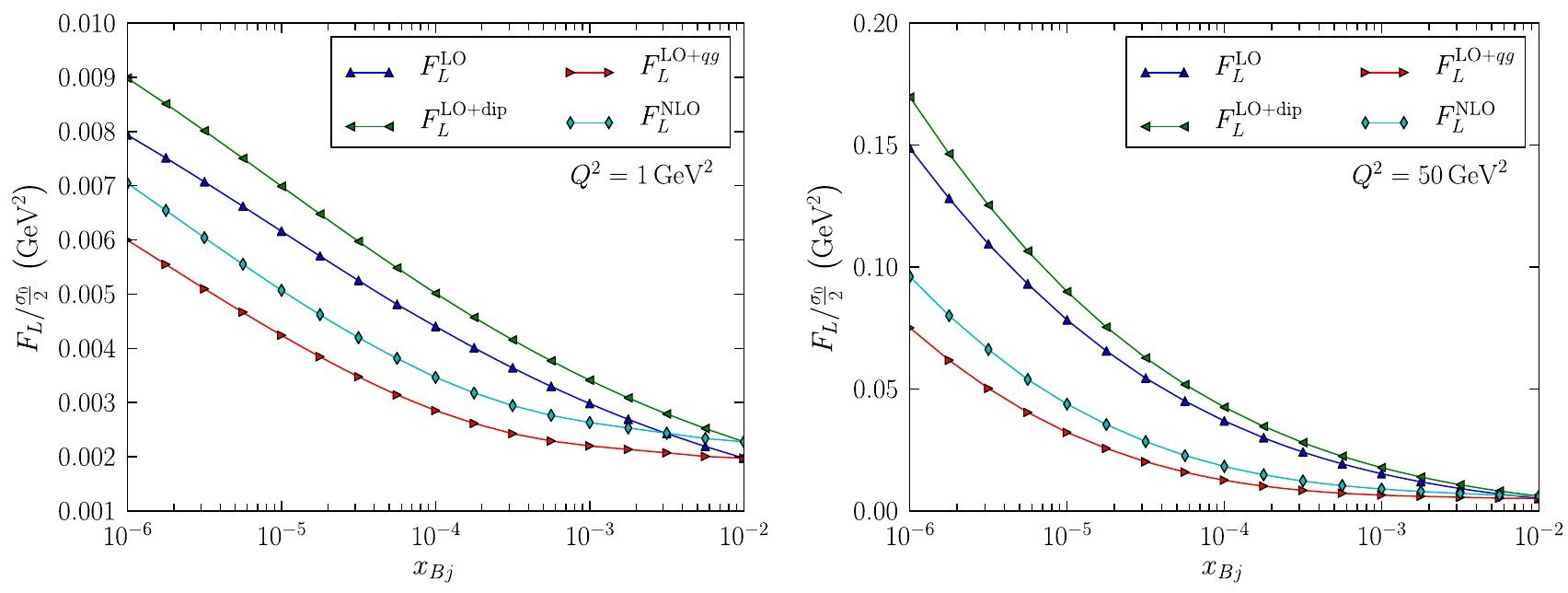

FIG. 3. LO and NLO contributions to $F_{L}$ as a function of $x_{B j}$ at $Q^{2}=1 \mathrm{GeV}^{2}$ (left) and $Q^{2}=50 \mathrm{GeV}^{2}$ (right) with $\alpha_{\mathrm{s}}=0.2$.
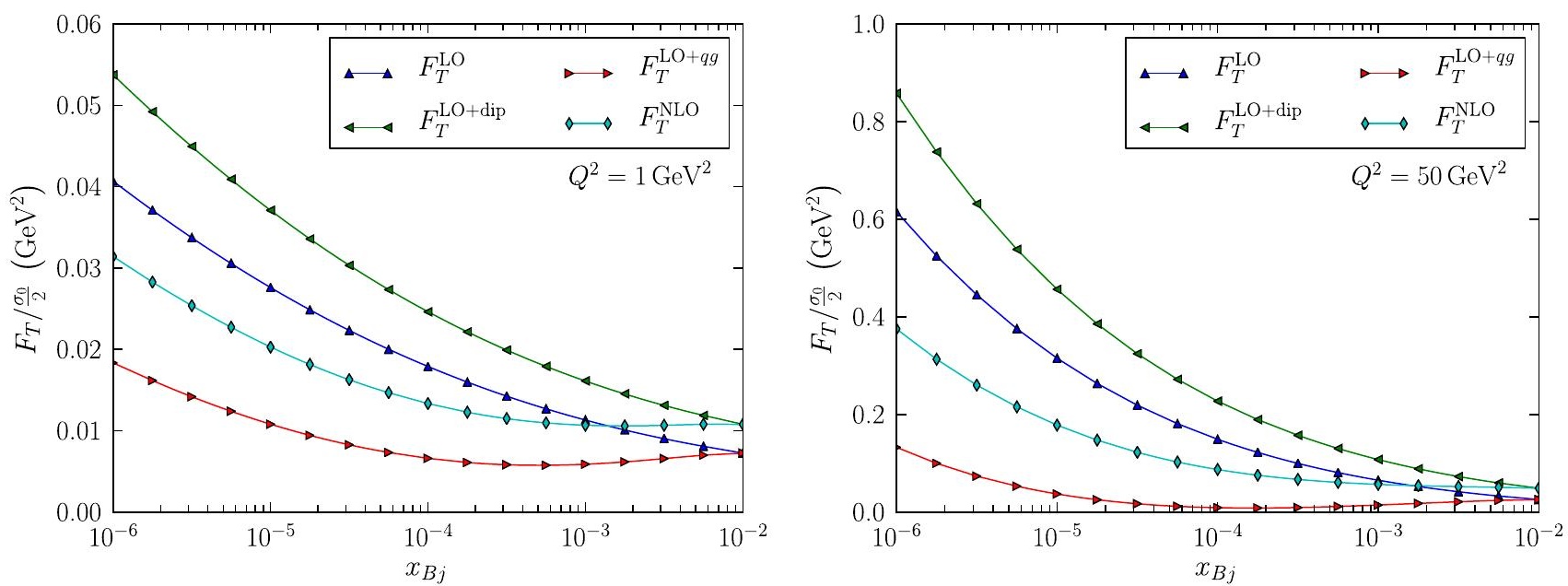

FIG. 4. LO and NLO contributions to $F_{T}$ as a function of $x_{B j}$ at $Q^{2}=1 \mathrm{GeV}^{2}$ (left) and $Q^{2}=50 \mathrm{GeV}^{2}$ (right) with $\alpha_{\mathrm{s}}=0.2$.

the dipole part which has an explicit integration over $z_{2}$. This would allow one to use, also in the dipole term, Wilson line operators at a rapidity scale which depends on the gluon momentum fraction, i.e. the invariant mass of the $q \bar{q} g$-state, in a way that is more consistent with the $q g$-part. The expressions we currently use restrict the kinematics to the regime of validity of the dipole picture $X<x_{0}$ for the $q g$-part but not for the dipole part. This leads to a sign change of the total NLO contribution as a function of $x_{B j}$ near $x_{0}$.

While the running of the strong coupling $\alpha_{\mathrm{s}}$ is in principle a subleading effect in a leading-order calculation, this effect has to be taken into account at next-to-leading order. To evaluate its importance here, we use the simple parent dipole prescription in which the coupling is given by

$$
\alpha_{\mathrm{s}}\left(\mathbf{x}_{01}^{2}\right)=\frac{4 \pi}{\beta_{0} \ln \left(\frac{4 C^{2}}{\mathbf{x}_{01}^{2} \Lambda_{\mathrm{QCD}}^{2}}\right)},
$$

with $\beta_{0}=\left(11 N_{\mathrm{c}}-2 n_{\mathrm{f}}\right) / 3$. The scaling parameter $C^{2}$ is taken to be $C^{2}=e^{-2 \gamma_{e}}$, as suggested in Refs. [31,32], and the coupling is frozen at the value 0.7 at large dipole sizes. When fitting the initial condition of the BK equation to data at leading order (see e.g. Refs. [3,4]), one usually uses instead the Balitsky prescription [33] for the running coupling and additionally takes $C^{2}$ as a fit parameter in order to obtain a slow enough evolution. However, in principle, the choice of the running coupling prescription is a higher-order effect, and thus the parent dipole prescription is equally well justified in a weak coupling sense. Also, on the phenomenological level, it has been shown $[8,10-$ 12] that the NLO corrections to the BK kernel slow down the evolution, and thus it is not a priori obvious which prescription will yield a good description of experimental data at the NLO level.

As stated before, our purpose here is not to achieve a fit to DIS data but to quantify the effect of the NLO corrections to the impact factor compared to previous 

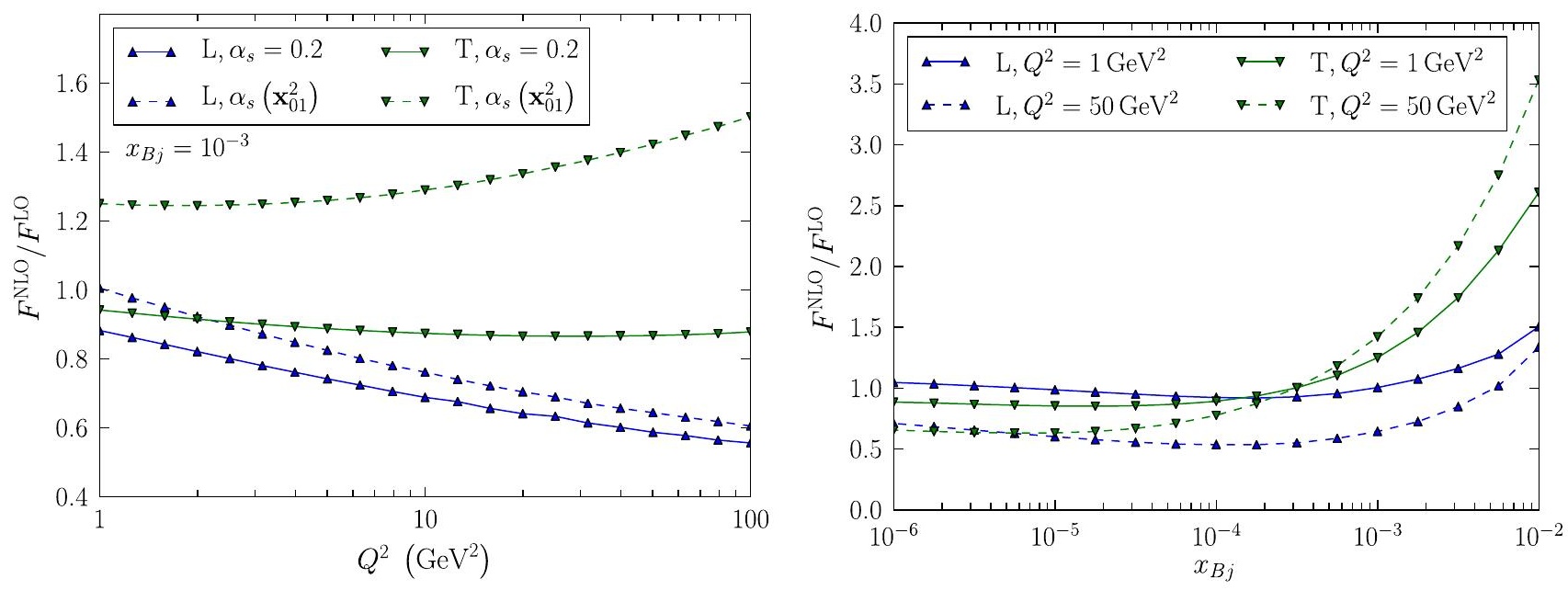

FIG. 5. Left: NLO/leading-order ratio for $F_{L}$ and $F_{T}$ as a function of $Q^{2}$ at $x_{B j}=10^{-3}$ with fixed (solid) and running (dashed) coupling. Right: NLO/leading-order ratio for $F_{L}$ and $F_{T}$ as a function of $x_{B j}$ at $Q^{2}=1 \mathrm{GeV}^{2}$ (solid) and $Q^{2}=50 \mathrm{GeV}$ (dashed) with running coupling.

LO calculations. Therefore, we show, in the left panel of Fig. 5, the NLO/LO ratio for $F_{L}$ and $F_{T}$ as a function of $Q^{2}$ at $x_{B j}=10^{-3}$ with fixed and running coupling. In the right panel, we show the same ratio as a function of $x_{B j}$ at $Q^{2}=1$ and $50 \mathrm{GeV}^{2}$ with running coupling. We see that for fixed coupling the net effect of the NLO corrections is to decrease the cross section. However, especially for a running coupling, this feature is reversed close to the initial rapidity scale $x_{B j} \approx x_{0}$. As discussed above, this is related to the fact that the negative NLO corrections related to BK evolution vanish in this limit while the positive ones in the dipole term do not, indicating a strong dependence on the details of the factorization scheme. While this is a transient effect that does not alter the asymptotic high energy behavior, treating it carefully will be important for an attempt to describe experimental data.

\section{OUTLOOK}

In conclusion, we have in this paper evaluated, for the first time, the total DIS cross section in the dipole picture with an impact factor derived at NLO accuracy. We developed a factorization procedure to resum the leading high energy logarithms into a BK renormalization group evolution of the target, in line with recent developments for single inclusive cross sections. We showed that this procedure leads to physical, well-behaved expressions for the cross sections with, however, large transient effects in the region close to the limit of validity of the eikonal approximation. With the caveat of understanding these transient effects, there is a good perspective for a comparison with experimental data. In order to achieve this at consistent NLO accuracy, the impact factors studied here must be combined with a solution of the NLO BK equation [12] or at least a collinearly resummed version of the LO equation [10,11]. A major missing theoretical ingredient that is needed for a more detailed comparison with data is to work out the corresponding impact factor for massive quarks. This should in principle be a straightforward, if laborious, extension of the existing calculation for massless quarks.

\section{ACKNOWLEDGMENTS}

We thank G. Beuf for sharing and discussing the results of Ref. [28] before publication, R. Paatelainen for discussions, and $\mathrm{H}$. Mäntysaari for sharing his BK evolution code. This work has been supported by the Academy of Finland, Projects No. 273464 and No. 303756 and by the European Research Council, Grant No. ERC-2015-CoG681707.
[1] I. Balitsky, Operator expansion for high-energy scattering, Nucl. Phys. B463, 99 (1996).

[2] Y. V. Kovchegov, Small-x F2 structure function of a nucleus including multiple pomeron exchanges, Phys. Rev. D 60, 034008 (1999).
[3] J. L. Albacete, N. Armesto, J. G. Milhano, P. Quiroga-Arias, and C. A. Salgado, AAMQS: A non-linear QCD analysis of new HERA data at small-x including heavy quarks, Eur. Phys. J. C 71, 1705 (2011). 
[4] T. Lappi and H. Mäntysaari, Single inclusive particle production at high energy from HERA data to protonnucleus collisions, Phys. Rev. D 88, 114020 (2013).

[5] T. Lappi and R. Paatelainen, The one loop gluon emission light cone wave function, Ann. Phys. (Amsterdam) 379, 34 (2017).

[6] A. Ayala, M. Hentschinski, J. Jalilian-Marian, and M. E. Tejeda-Yeomans, Spinor helicity methods in high-energy factorization: Efficient momentum-space calculations in the color glass condensate formalism, Nucl. Phys. B920, 232 (2017).

[7] I. Balitsky and G. A. Chirilli, Next-to-leading order evolution of color dipoles, Phys. Rev. D 77, 014019 (2008).

[8] T. Lappi and H. Mäntysaari, Direct numerical solution of the coordinate space Balitsky-Kovchegov equation at next to leading order, Phys. Rev. D 91, 074016 (2015).

[9] G. Beuf, Improving the kinematics for low-x QCD evolution equations in coordinate space, Phys. Rev. D 89, 074039 (2014).

[10] E. Iancu, J. D. Madrigal, A. H. Mueller, G. Soyez, and D. N. Triantafyllopoulos, Resumming double logarithms in the QCD evolution of color dipoles, Phys. Lett. B 744, 293 (2015).

[11] E. Iancu, J. D. Madrigal, A. H. Mueller, G. Soyez, and D. N. Triantafyllopoulos, Collinearly-improved BK evolution meets the HERA data, Phys. Lett. B 750, 643 (2015).

[12] T. Lappi and H. Mäntysaari, Next-to-leading order BalitskyKovchegov equation with resummation, Phys. Rev. D 93, 094004 (2016).

[13] G. A. Chirilli, B.-W. Xiao, and F. Yuan, One-Loop Factorization for Inclusive Hadron Production in pA Collisions in the Saturation Formalism, Phys. Rev. Lett. 108, 122301 (2012).

[14] G. A. Chirilli, B.-W. Xiao, and F. Yuan, Inclusive hadron productions in pA collisions, Phys. Rev. D 86, 054005 (2012).

[15] A. M. Stasto, B.-W. Xiao, and D. Zaslavsky, Towards the Test of Saturation Physics beyond Leading Logarithm, Phys. Rev. Lett. 112, 012302 (2014).

[16] Z.-B. Kang, I. Vitev, and H. Xing, Next-to-Leading Order Forward Hadron Production in the Small- $x$ Regime: Rapidity Factorization, Phys. Rev. Lett. 113, 062002 (2014).

[17] A. M. Staśto, B.-W. Xiao, F. Yuan, and D. Zaslavsky, Matching collinear and small $x$ factorization calculations for inclusive hadron production in $p A$ collisions, Phys. Rev. D 90, 014047 (2014).
[18] T. Altinoluk, N. Armesto, G. Beuf, A. Kovner, and M. Lublinsky, Single-inclusive particle production in protonnucleus collisions at next-to-leading order in the hybrid formalism, Phys. Rev. D 91, 094016 (2015).

[19] K. Watanabe, B.-W. Xiao, F. Yuan, and D. Zaslavsky, Implementing the exact kinematical constraint in the saturation formalism, Phys. Rev. D 92, 034026 (2015).

[20] B. Ducloué, T. Lappi, and Y. Zhu, Single inclusive forward hadron production at next-to-leading order, Phys. Rev. D 93, 114016 (2016).

[21] E. Iancu, A. H. Mueller, and D. N. Triantafyllopoulos, CGC factorization for forward particle production in protonnucleus collisions at next-to-leading order, J. High Energy Phys. 12 (2016) 041.

[22] B. Ducloué, T. Lappi, and Y. Zhu, Implementation of NLO high energy factorization in single inclusive forward hadron production, Phys. Rev. D 95, 114007 (2017).

[23] I. Balitsky and G. A. Chirilli, Photon impact factor in the next-to-leading order, Phys. Rev. D 83, 031502 (2011).

[24] G. Beuf, NLO corrections for the dipole factorization of DIS structure functions at low x, Phys. Rev. D 85, 034039 (2012).

[25] R. Boussarie, A. V. Grabovsky, L. Szymanowski, and S. Wallon, Impact factor for high-energy two and three jets diffractive production, J. High Energy Phys. 09 (2014) 026.

[26] R. Boussarie, A. V. Grabovsky, L. Szymanowski, and S. Wallon, On the one loop $\gamma^{(*)} \rightarrow q \bar{q}$ impact factor and the exclusive diffractive cross sections for the production of two or three jets, J. High Energy Phys. 11 (2016) 149.

[27] G. Beuf, Dipole factorization for DIS at NLO: Loop correction to the photon to quark-antiquark light-front wave-functions, Phys. Rev. D 94, 054016 (2016).

[28] G. Beuf, Dipole factorization for DIS at NLO: Combining the $q \bar{q}$ and $q \bar{q} g$ contributions, Phys. Rev. D 96, 074033 (2017).

[29] L. Motyka and A. M. Stasto, Exact kinematics in the small x evolution of the color dipole and gluon cascade, Phys. Rev. D 79, 085016 (2009).

[30] L. D. McLerran and R. Venugopalan, Computing quark and gluon distribution functions for very large nuclei, Phys. Rev. D 49, 2233 (1994).

[31] Y. V. Kovchegov and H. Weigert, Triumvirate of running couplings in small-x evolution, Nucl. Phys. A784, 188 (2007).

[32] T. Lappi and H. Mäntysaari, On the running coupling in the JIMWLK equation, Eur. Phys. J. C 73, 2307 (2013).

[33] I. Balitsky, Quark contribution to the small- $x$ evolution of color dipole, Phys. Rev. D 75, 014001 (2007). 